\title{
Über einen weiteren synthetischen Versuch in der Gentisinreihe
}

von

St. v. Kostanecki und J. Tambor.

Aus dem chemischen Laboratorium der Universität in Bern.

(Vorgelegt in der Sitzung am 14. November 1895.)

Wie wir vor $1 \frac{1}{2}$ Jahren mitgetheilt haben, ${ }^{1}$ bildet sich Gentisin, wenn man das durch Condensation von Hydrochinoncarbonsäure und Phloroglucin entstandene 1-, 3-, 7-Trioxyxanthon methylirt.

Die Constitution des Gentisins ergab sich indessen noch nicht mit voller Sicherheit aus der obigen Synthese. Von den drei theoretisch möglichen Gentisin-Formeln:
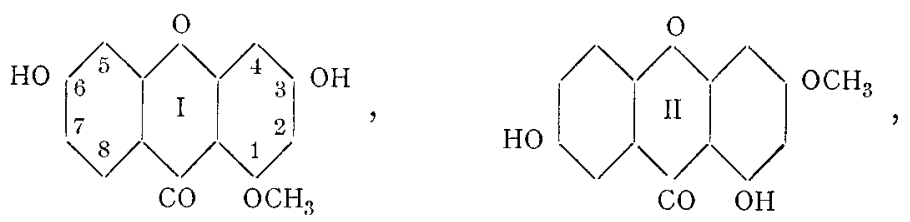

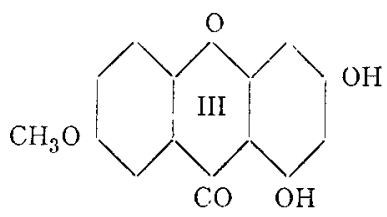

war allerdings die erste ausgeschlossen, da bei den Oxyxanthonen nach Dreher und dem Einen von uns ${ }^{2}$ das in der

1 Monatshefte für Chemie, 15, 1.

$\because$ Berichte der Deutschen chem. Gesellsch., 26, 71 . 
Stellung 1 befindliche Hydroxyl unter den gewöhnlichen Bedingungen nicht alkylirbar ist; die beiden anderen Formeln erschienen jedoch als gleichberechtigt.

Wir versuchten nun, da wir inzwischen eine bequeme Darstellungsweise für die Hydrochinonmonomethyläthercarbonsäure von der Constitution

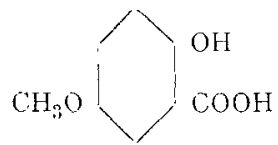

gefunden hatten, die Frage nach der wahren Formel des Gentisins auf synthetischem Wege zu lösen.

Durch Paarung dieser Säure mit Phloroglucin wäre nàmlich die Bildung des 1-, 3-, 7-Trioxyxanthon-7-Methyläthers (Formel III) zu erwarten, dessen Vergleich mit dem natürlichen Gentisin die Entscheidung zwischen den beiden Formeln II und III liefern konnte.

Der Versuch ergab, wie weiter unten ausgeführt werden soll, ein zweideutiges Resultat, so dass die Constitutionsformel des Gentisins auch heute noch zu ermitteln bleibt. Immerhin besitzen die von uns gesammelten Erfahrungen nach einigen Richtungen ein gewisses Interesse, wesshalb wir sie in aller Kurze hier mittheilen.

Die

\section{Hydrochinonmonomethyläthercarbonsäure}

(Netamethoxysalicylsäure)

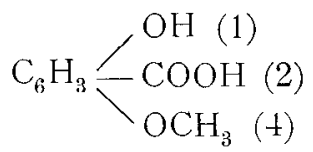

ist von $\mathrm{Körner}$ und Berton $\mathrm{i}^{1}$ durch Einwirkung von Kohlensäure auf Hydrochinonmonomethyläthernatrium und von Tiemann und Müller bei der Oxydation von Acet-m-Methoxysalicylaldehyd erhalten worden.

1 Berichte der Deutschen chem. Gesellschaft, 14, $8+8$.

2 Berichte der Deutschen chem. Gesellschaft, 14, 1997. 
Sie lässt sich leicht darstellen, wenn man Hydrochinoncarbonsäure (1 Mol.) mit 2 Moleküle Alkalı und etwas mehr als 2 Moleküle Methyljodid am Rückflusskühler einige Stunden lang auf dem Wasserbade erhitzt. Es wird hiebei nur das in der $m$-Stellung zur Carboxylgruppe befindliche Hydroxyl methylirt, während diejenige Hydroxylgruppe, welche in der $o$-Stellung zum Carboxyl steht, intact bleibt, ganz ebenso, wie dies beim Alkyliren der $\beta$-Resorcylsüure der Fall ist. ${ }^{1}$ Der nach dem Alkyliren eingedampfte Kolbeninhalt wird mit verdunnter Natronlauge aufgenommen. filtrirt und die Säure mit Salzsäure gefällt. Durch Umkrystallisiren aus heissem Wasser lässt sie sich von der etwa unangegriffenen, viel leichter löslichen Hydrochinoncarbonsäure befreien. Nach nochmaligem Umkrystallisiren aus Benzol erhält man sie in langen, weissen Nadeln, die bei $150^{\circ}$ schmelzen und mit Eisenchlorid eine blaue Färbung geben.

$0.2050 \mathrm{~g}$ Substanz lieferten $0.4330 \mathrm{~g}$ Kohlendiuxyd und () $0906 \mathrm{~g}$ Wasser.

$(0 \cdot 2012 g$ Substanz lieferten $0.42+4 g$ Kohlendioxyd und $0 \cdot 0880 \mathrm{~g}$ Wasser.

\begin{tabular}{|c|c|c|}
\hline \multicolumn{2}{|c|}{ Gefunden } & \multirow{2}{*}{$\begin{array}{c}\text { Berechnet für } \\
\mathrm{C}_{9} \mathrm{H}_{10} \mathrm{O}_{1}\end{array}$} \\
\hline I & II & \\
\hline$\ldots .57 \cdot 56$ & $57 \cdot 50$ & $57 \cdot 1+\%$ \\
\hline$\ldots \ldots+90$ & $4 \cdot 82$ & 4.76 \\
\hline
\end{tabular}

1 Tiemann und Parisius, Ber. d. Deutschen chem. Gesellsch 13, 2354. Kostanecki und Tambor, Ber. d. Deutschen chem. Gesellsch. 28, 2308

Ebenso leicht entsteht die Hydrochinonmonoathyläthercarbonsäure, wenn man die Hydrochinoncarbonsaure mit Athylbromid und Kalihydrat auf dem Wasserbade erhitzt. Sie krystallisirt in weissen Nadeln vom Schmelzpunkt $164^{\circ}$.

$0 \cdot 1710 \mathrm{~g}$ Substanz gaben $0.3737 \mathrm{~g}$ Kohlendioxyd und $0.0845 \mathrm{~g}$ Wasser. $0.2192 \mathrm{~g}$ Substanz gaben $0 \cdot 4780 \mathrm{~g}$ Kohlendioxyd und $0.1122 \mathrm{~g}$ Wasser.

In 100 Theilen:

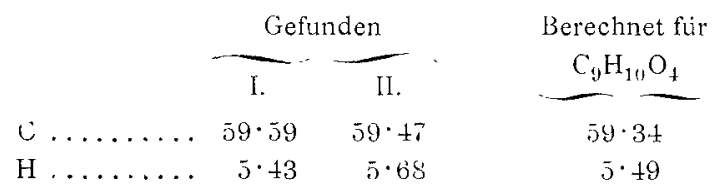


Die Hydrochinonmonomethyläthercarbonsäure wurde nun mit Phloroglucin (in äquimolecularen Mengen) und mit der zur Lösung erforderlichen Menge Essigsäureanhydrid in kleinen Antheilen aus einer Retorte vorsichtig destillirt. Die Reaction geht hier bedeutend glatter von statten, als dies bei der Paarung von Hydrochinoncarbonsäure mit Phloroglucin der Fall ist.

Allmälig sammelt sich im Halse der Retorte ein gelblich gefärbtes, festes Sublimat, welches sich durch verdünnte Natronlauge in zwvei Theile zerlegen lässt. Der in grösserer Menge entstandene, alkaliunlösliche Theil war intensiv gefärbt und stellte das Natriumsalz einer Verbindung vor, die durch Übergiessen des Salzes mit verdünnter Salzsäure in Freiheit gesetzt werden konnte. Dieselbe wurde aus viel Alkohol oder besser aus Eisessig umkrystallisirt. Wir erhielten so gelblich gefärbte, breite Nadeln rom Schmelzpunkt $167^{\circ}$, deren Analyse Zahlen ergab, die scharf auf die Formel eines Trioxyxanthondimethyläthers stimmen.

$0.2054 \mathrm{~g}$ Substanz lieferten $0.4969 \mathrm{~g}$ Kohlendioxyd und $0.0862 g$ Wasser.

$0.1997 \mathrm{~g}$ Substanz lieferten $0.4838 \mathrm{~g}$ Kohlendioxyd und $0.0844 g$ Wasser.

In 100 Theilen:

\begin{tabular}{|c|c|c|c|}
\hline & Gef & den & Berechnet für \\
\hline & $\mathrm{I}$ & II & $\mathrm{C}_{15} \mathrm{H}_{1}, \mathrm{O}_{5}$ \\
\hline C & $65 \cdot 97$ & $66 \cdot 07$ & $66 \cdot 17$ \\
\hline $\mathrm{H}$. . & $4 \cdot 66$ & $4 \cdot 66$ & $4 \cdot 41$ \\
\hline
\end{tabular}

Wenn auch die Eigenschaften dieses Körpers keinen Zweifel übrig liessen, dass hier der von Schmidt und dem Einen von uns ${ }^{1}$ beschriebene Gentisinmonomethyläther (1-, 3-, 7-Trioxyxanthon-3-, 7-Dimethyläther)

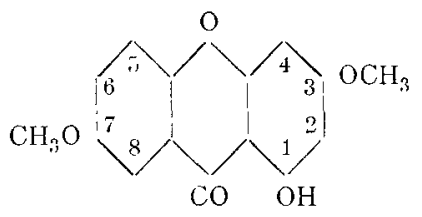

1 Monatshefte fur Chemie, 12, 318. 
vorlag, so haben wir doch behufs ganz sicherer Identificirung noch seine Acetylverbindung dargestellt und auch bei diesem Derivate rollständige Übereinstimmung mit dem früheren Präparate festgestellt.

Unverhofft sind wir somit zu einer relativ leichten synthetischen Gewinnung des 1-, 3-, 7-Trioxyxanthon-3-, 7-Dimethyläthers gelangt, die mit unseren früheren Arbeiten über dus Gentisin in besten Einklang gebracht werden kann.

Bei der Destillation wird offenbar entweder aus der Hydrochinonmonomethyläthercarbonsäure oder wahrscheinlicher aus dem gebildeten 1-,3-,7-Trioxyxanthonmonomethyläther Methylalkohol abgespalten, der bei der hohen Temperatur auf ein zweites Molekül des Äthers methylirend einwirkt. Dementsprechend haben wir neben dem 1-,3-,7-Trioxyxanthonmonomethyläther, welcher sich mit dem Gentisin als identisch erwies, das entmethylirte Product - das 1-, 3-, 7-Trioxyxanthon (Gentisein) - wenn auch nur in geringer Menge, nachweisen können.

Das oben erwähnte alkalische Filtrat ergab beim Ansäuern mit Salzsäure einen voluminösen Niederschlag, der Thonerdebeize schwach gelb anfärbte, was schon für die Anwesenheit des 1-,3-,7-Trioxyxanthons sprach. Um dasselbe zu isoliren, wurde der scharf getrocknete Niederschlag mit Essigsäureanhydrid und entwässertem essigsauren Natron kurze Zeit gekocht und das entstandene Acetylproduct mit heissem Alkohol ausgezogen. Es hinterblieb ein krystallinischer, weisser Rückstand, der aus Eisessig in blendend weissen Nadeln krystallisirte, die den von dem Einen von uns $^{1}$ für das Triacetylgentiseïn angegebenen Schmelzpunkt $226^{\circ}$ besassen.

Der grösste Theil des rohen Acetylproductes war in warmem Alkohol leicht löslich. Beim Einengen der Lösung schieden sich rosettenförmig gruppirte, voluminöse, weisse Nadelchen ab, die so lange aus Alkohol umkrystallisirt wurden, bis sie ein einheitliches Aussehen besassen. Durch den Schmelzpunkt $\left(195^{\circ}\right)$ und durch die bei der Analyse erhaltenen Zahlen

1 Monatshefte für Chemie, 12, 205. 
charakterisirt sich diese Verbindung als das von Hlasiwetz und Hiabermann ${ }^{1}$ beschriebene Diacetylgentisin.

$0.2048 g$ Substanz lieferten $0 \cdot 4764 g$ Kohlendioxyd und $0 \cdot 0739 g$ Wasser.

In 100 Theilen:

\begin{tabular}{|c|c|}
\hline Gefunden & $\begin{array}{c}\text { Berechnet fuir } \\
\mathrm{C}_{18} \mathrm{H}_{11} \mathrm{O}_{7}\end{array}$ \\
\hline $63 \cdot 44$ & $63 \cdot 15$ \\
\hline $4 \cdot 01$ & $4 \cdot 09$ \\
\hline
\end{tabular}

Beim Kochen mit verdünnter Natronlauge ging das Diacetylgentisin mit gelber Farbe in Lösung. Das durch Zusatz von Salzsäure ausgefällte Gentisin wurde aus Alkohol umkrystallisirt. Die hiebei resultirenden gelben Nädelchen gaben mit Natriumamalgam die Gentisin-Reaction und schmolzen bei $263^{\circ}$. (Schmelzpunkt des natürlichen Gentisins 267\%.)

Wir glauben demnach die Anwesenheit des Gentisins in dem Reactionsproducte aus Hydrochinonmonomethyläthercarbonsäure und Phloroglucin nachgewiesen zu haben, wollen aher nicht behaupten, dass dasselbe wirklich nach der Gleichung

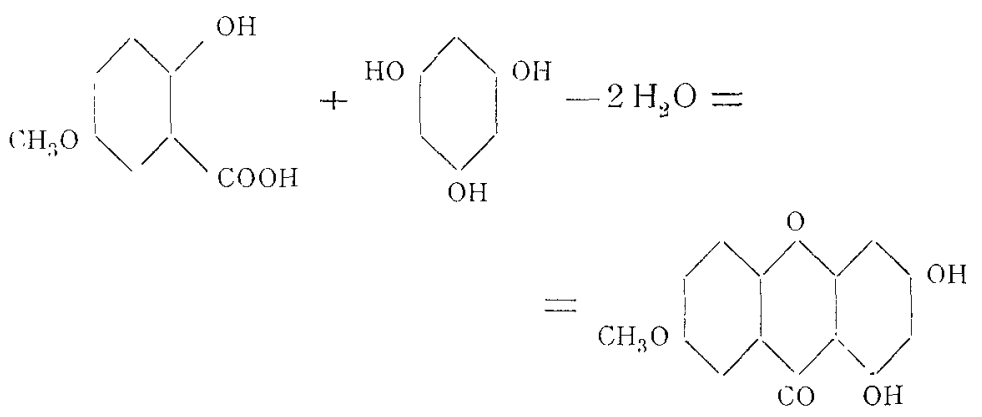

entstanden ist. Man kann nämlich sehr wohl annehmen, und diesen Einwand können wir durch kein Argument beseitigen, das Gentisin sei in Folge der Methylirung des 1-, 3-, 7-Trioxyxanthons als secundäres Product aufgetreten, während das nach der obigen Gleichung entstandene Product Isogentisin sei und

1 Lrebig's Annalen, 175, 75. 
durch weitere Methylirung in den 1-, 3-, 7-Trioxyxanthon-3-, 7 -Dimethyläther übergegangen sei. Sobald wir eine Wanderung der Methylgruppe bei der Reaction constatirt hatten, war es klar, dass die Lösung der Frage, ob hier ein Gentisin oder Isogentisin entstanden sei, für die Feststellung der Constitutionsformel des Gentisins bedeutungslos geworden ist. Wir haben trotzdem die mühsame Untersuchung durchgeführt, weil hier die von Nessler und dem Einen ${ }^{1}$ von uns angegebene, allgemeine Darsteliungsmethode der Oxyxanthone zum ersten Nale für die Gewinnung der Oxyxanthonäther zur Anwendung kam.

$\mathrm{Da}$ in der Gentisinreihe die hier auftretenden Nethyläther schon bekannt waren, so war es möglich, trotz der geringen Mengen, die verschiedenen Reactionsproducte von einander zu trennen und $\mathrm{zu}$ charakterisiren.

In einem anderen Falle könnten die hier beobachteten Verschiebungen der Methylgruppen, wenn sie a priori nicht erwartet würden, die Untersuchung der Reaction noch viel mehr erschweren und eventuell zu Fehlschlüssen führen.

1 Ber. d. Deutschen chem. Ges. 24, 1891 und 3981. 